\title{
Niveles de fondo geoquímico e influencia del marco geológico en las concentraciones edafogeoquímicas de base de suelos seleccionados de Castilla-La Mancha
}

\author{
Geochemical background levels and influence of the geological setting \\ on pedogeochemical baseline concentrations of trace elements \\ in selected soils of Castilla-La Mancha (Spain)
}

\author{
R. Jiménez Ballesta ${ }^{1}$, P. Conde Bueno ${ }^{2}$, J.A. Martín Rubí ${ }^{2}$ R. García Giménez ${ }^{1}$
}

RESUMEN

La determinación de las concentraciones geoquímicas de base es necesaria para la legislación ambiental y toma de decisiones, especialmente en la evaluación de suelos contaminados.

Aunque la terminología sobre concentraciones geoquímicas (variaciones naturales en la concentración en el medio ambiente de superficie), aparece en los estudios geoquímicos y programas internacionales, probablemente, nunca ha sido bien definida.

Para contestar a cuestiones como ¿cuál es la concentración natural de una serie de elementos traza en suelos y rocas de Castilla-La Mancha?, se determinan el fondo geoquímico y las concentraciones edafogeoquímicas de base, a la vez que se calculan los valores umbrales de los mismos. Todos los elementos traza muestran concentraciones promedio similares con los valores de referencia para los suelos de otras zonas de España (Galicia, Andalucía, Madrid, etc.), la Unión Europea y el mundo. Los patrones de distribución de las concentraciones de los elementos son principalmente influenciados por la naturaleza geoquímica de la roca.

Palabras clave: Elementos traza, fondo geoquímico, concentraciones geoquímicas de base en suelos, valores de referencia, Castilla-La Mancha.

\begin{abstract}
Geochemical baselines are needed for environmental legislation and political decision-making, especially in the assessment of contaminated soil.

Although the term «geochemical baseline» appears in the international geochemical studies and programs, it has never been well defined. A geochemical baseline for an element refers to its natural variations in concentration in the superficial environment.

In order to answer questions such as «what is the natural concentration of trace elements in rocks and soils of Castilla-La Mancha region?» this work seeks to establish the geochemical background, baseline pedo-geochemical concentrations and reference values for the Castilla-La Mancha region (Central Spain). Trace element concentrations in the study area were similar to those analysed in other areas of Spain and the world. Their baseline concentrations depend mainly on the geochemical characteristics of the bed rocks.
\end{abstract}

Key words: Trace elements, geochemical background, baseline pedo-geochemical concentration, reference values, Castilla-La Mancha.

\footnotetext{
1 Departamento de Geología y Geoquímica. Facultad de Ciencias. Universidad Autónoma de Madrid. Campus Cantoblanco. 28049 Madrid. Email: raimundo.jimenez@uam.es

2 Instituto Geológico y Minero de España. Departamento de Infraestructura Geocientífica y Servicios. Área de Laboratorios y Servicios, c/ La Calera, 1. 28760 Tres Cantos. Madrid.
} 


\section{Introducción}

El suelo es un recurso natural muy importante para el desarrollo sostenible. El estudio de su calidad (especialmente en Europa), normalmente se focaliza en relación a su posible contaminación. Concretamente, sobre la determinación de la concentración natural base de los contaminantes y sus potenciales efectos adversos.

La evaluación y control de los suelos contaminados requiere disponer de datos, a fin de salvaguardar la calidad humana, animal y ambiental. En este sentido, las concentraciones de elementos traza en suelos deben ser incluidas en cualquier evaluación de la calidad ambiental del suelo, ya que un déficit o exceso de los mismos puede afectar el crecimiento vegetal y animal y/o la salud humana (Fergusson, 1990 y Baize \& Sterckeman, 2001).

Las concentraciones de fondo geoquímico natural deben representar concentraciones elementales en los suelos sin influencia humana. Concentraciones de base geoquímica, en cambio, corresponden a una concentración específica para una zona y tiempo determinados (Kabata-Pendias et al., 1984 y Kabata-Pendias $\&$ Pendias, 1992). Por ello, se pueden establecer tres tipos de niveles de fondo: a) Niveles naturales en rocas: concentraciones naturales de elementos presentes en las rocas; $b$ ) Niveles naturales en el medio ambiente: concentraciones naturales de elementos presentes en el medio ambiente (suelo/roca), sin influencia del ser humano; c) Niveles antropogénicos: concentraciones de elementos presentes en el medio ambiente debidas a fuentes no localizadas de origen humano (por ejemplo, agrícolas, industriales, etc.).

En cualquier caso, sea cual sea la denominación, la determinación de las concentraciones geoquímicas ambientales de base son necesarias para evaluar el estado actual del medio ambiente y proporcionar directrices y normas de calidad para la legislación ambiental, así como la toma de decisiones de gestión, especialmente en la evaluación de suelos contaminados (Salminen \& Tarvainen, 1997). Por lo tanto, para evaluar la contaminación del suelo es importante determinar las concentraciones de fondo (o concentraciones de base geoquímica) que, según Kabata-Pendias \& Pendias (1992) y Chen et al. (1999) debe realizarse de acuerdo a las clases de suelo y propiedades. Una crítica de los métodos utilizados para las determinaciones de fondo se debe a Reimann et al. (2005).

Según Bak et al. (1997) pocos países cuentan con información detallada sobre el contenido de metales pesados de las zonas contaminadas. Pero se han rea- lizado numerosos esfuerzos en identificar los niveles de base, antes y después de la contaminación de origen antropogénico (Thornton, 1981; Fleischhauer \& Korte, 1990; Mckeague \& Wolynetz, 1997; Lee et al., 1997; Ma et al., 1997; Del Castillo et al., 2003; Nsouli et al., 2004; etc.).

En el caso español la base de datos sobre concentraciones geoquímicas de elementos traza varía entre las diferentes regiones. Así, se han llevado a cabo estudios para evaluar la abundancia y distribución de elementos traza tóxicos en los suelos de varias comunidades y regiones españolas (IHOBE, 1993; Junta de Residus, 1998; Junta de Andalucía, 1999; Junta de Castilla y León, 1999; De Miguel et al., 2002; Del Castillo et al., 2003; Martínez Sánchez \& Pérez Sirvent, 2008; Conde et al., 2009; Macías \& Calvo de Anta, 2009; etc.). El Real Decreto 9/2005 (BOE 2005) propone la obtención de valores de referencia para metales.

En Castilla-La Mancha, actualmente, existen valores de referencia globales de elementos traza potencialmente tóxicos en suelos (Conde et al., 2009), pero no para distintos usos de la tierra, tales como la agricultura, desarrollo industrial, recreativo, etc. Con el ánimo de contribuir, por una parte, a evaluar la contaminación de los recursos naturales edáficos $\mathrm{y}$, por otra, al conocimiento de las variaciones existentes en la concentración de elementos traza en rocas y suelos, el objetivo fundamental del presente estudio (que es continuación del inmediato anteriormente citado) es establecer las premisas necesarias para determinar y presentar una propuesta de los valores genéricos de referencia de un amplio espectro de elementos traza $(\mathrm{Sc}, \mathrm{V}, \mathrm{Cr}, \mathrm{Co}, \mathrm{Ni}, \mathrm{Cu}, \mathrm{Zn}$, $\mathrm{Ga}, \mathrm{Ge}, \mathrm{As}, \mathrm{Se}, \mathrm{Br}, \mathrm{Rb}, \mathrm{Sr}, \mathrm{Y}, \mathrm{Zr}, \mathrm{Nb}, \mathrm{Mo}, \mathrm{Ag}, \mathrm{Cd}$, $\mathrm{Sn}, \mathrm{Sb}, \mathrm{I}, \mathrm{Cs}, \mathrm{Ba}, \mathrm{La}, \mathrm{Ce}, \mathrm{Nd}, \mathrm{Sm}, \mathrm{Yb}, \mathrm{Hf}, \mathrm{Ta}, \mathrm{W}$, $\mathrm{Tl}, \mathrm{Pb}, \mathrm{Bi}$, Th y U), en suelos de Castilla-La Mancha. El fin último es obtener criterio para identificar si un suelo está contaminado.

\section{Material y métodos}

\section{Área de estudio y procedimiento de muestreo}

Castilla-La Mancha, tiene una gran variedad litológica: calizas, margas, granitos, basaltos, areniscas, etc. Desde el punto de vista botánico, se puede observar una variadísima tipología de especies y formaciones: bosques, de praderas, etc., si bien una parte sustancial del territorio $(>50 \%)$, se dedica a 
uso agrícola. La región se caracteriza por un clima mediterráneo que varía con la latitud, la altitud y continentalidad. Si a esta variedad se suma una gran diversidad de posiciones geomorfológicas, no es extraño encontrar variados tipos de suelos Así, según los criterios de la FAO (2006), existen Leptosoles, Vertisoles, Fluvisoles, Kastanozems, Gypsisoles, Calcisoles, Acrisoles, Luvisoles, Umbrisols, Cambisoles y Regosoles y, en en menor medida, Antrosoles, Technosoles, Solonchaks, Chernozems, Histosoles, Phaeozems y Arenosoles. Más raros son Solontezs, Podsoles, Nitisoles, Ferralsoles, Planosoles, Stagnosols, Albeluvisoles, Alisoles y Lixisoles. No hay Cryosoles, Andosoles, Plintosoles ni Durisoles. De acuerdo con Soil Taxonomy (2006) aparecen: Entisoles, Inceptisoles, Alfisoles, Molisoles y en menor medida, Aridisoles, Histosoles, Vertisoles y Ultisoles, mientras que Podsoles son raros. Ni Andosoles (a pesar de la existencia de material volcánico vario), ni Oxisoles ni Gelisoles fueron encontrados. Por lo tanto, se puede decir que Castilla-La Mancha tiene una gran diversidad de suelos.

Las muestras (tabla 1) se seleccionaron de los suelos más predominantes y diversidad de materiales de partida: Cambisoles, Leptosoles, Regosoles, Calcisoles, Vertisoles, Umbrisols y Luvisoles y, con el ánimo de responder a las siguientes preguntas: a) ¿cuál es la concentración natural en rocas, es decir, fondo geoquímico?; b) ¿cuál es la concentración natural de los suelos, es decir, concentración edafogeoquímica, aunque en un trabajo previo (Conde et al., 2009) se había demostrado que ambas poblaciones no son diferentes? En cada lugar se muestreó el horizonte de superficie (A), el horizonte subsuperficial (B o C) y el horizonte de profundidad (C o R).

\section{Métodos analíticos}

Las muestras de suelo fueron secadas al aire y, previa trituración con un rodillo de madera, tamizada a $<2 \mathrm{~mm}$. Posteriormente se trituraron en un mortero de ágata. La fracción $<63$ micras se separó para el análisis químico. Los análisis químicos se han realizado en los laboratorios del IGME. El contenido total de los elementos traza se determinó mediante XRF, utilizando el Programa Pro Trace, con equipo MAGIX de Panalytical y con pastilla prensada de 8 gramos compactada con Elvacite. Algunos elementos se encuentran por debajo del límite de detección, por lo que sus valores son considerados como una estimación preliminar.

\section{Resultados y discusión}

Los valores de concentración medios de los elementos traza analizados, clasificados por horizonte superior (A), medio (B o C) e inferior ( $\mathrm{C}$ o R), se resumen en la tabla 2.

Trece de los elementos analizados ( $\mathrm{Co}, \mathrm{Ge}, \mathrm{Se}$, Mo, Ag, Cd, Sb, Yb, Hf, Ta, W, Tl y Bi) presentaron valores iguales o por debajo del límite de detección del método analítico utilizado, por lo que se imponen ciertas limitaciones de interpretación.

Se entiende que nivel de fondo es la concentración normal de uno o varios elementos químicos en el medio ambiente. Estas concentraciones naturales de fondo, en el caso de los suelos, pueden haber sido alteradas significativamente por efecto de numerosas actividades antropogénicas, tales como en las que se practica el uso de fertilizantes, la minería, el uso industrial. El fondo geoquímico podría ser definido como el contenido en elementos traza que presenta un suelo sin la influencia de la actividad humana (Vidal et al., 2004).

Según Tack et al. (1997) en el «ambiente natural», las concentraciones son las que se derivan exclusivamente de los procesos naturales. El «fondo real» se define como la concentración típica de un elemento que se encuentra en un terreno que no ha sido objeto de contaminación. En el caso de estudio, los resultados (datos de los horizontes C-R de la tabla 2), expresan los valores de fondo, que son similares a los de otras zonas y variados en función de la litología.

El término «concentración natural o concentración geoquímica de base», se refiere a la variación natural en la concentración de un elemento en el medio ambiente superficial (Salminen \& Tarvainen, 1997 y Salminen \& Gregorauskiene, 2000); puede indicar el contenido real de un elemento en el medio ambiente superficial en un punto dado y en un momento temporal (Salminen \& Gregorauskiene, 2000; Frattini et al., 2006 y Albanese et al., 2007). Incluye las concentraciones geogénicas naturales (natural de fondo) y la contribución antropogénica difusa de los suelos (Tarvainen \& Kallio, 2002; Cicchella et al., 2005; Frattini et al., 2006 y Albanese et al., 2007). Bajo esta perspectiva, los valores encontrados (datos de los horizontes A y B-C de la tabla 2) son, en promedio, similares a los valores de referencia para los suelos de otras zonas de España (Galicia, Andalucía, Madrid, etc.), la Unión Europea y el mundo.

Aunque la variación del suelo causada por procesos de génesis es un proceso natural, a veces intenso, las variaciones en las concentraciones de ele- 
Tabla 1.-Características generales de los suelos y rocas seleccionados

\begin{tabular}{|c|c|c|c|c|c|}
\hline $\begin{array}{l}\text { № de } \\
\text { perfil }\end{array}$ & $\begin{array}{l}\text { Tipo de suelo } \\
\text { (FAO, 2006) }\end{array}$ & Localización & $\begin{array}{l}\text { Roca } \\
\text { de partida }\end{array}$ & $\begin{array}{l}\text { Vegetación/ } \\
\text { Uso }\end{array}$ & Coordenadas \\
\hline 1 & $\begin{array}{l}\text { Leptic Regosol } \\
\text { (Gypsiric, Siltic) }\end{array}$ & $\begin{array}{l}\text { Ontigola } \\
\text { (Toledo) }\end{array}$ & Yesos & Erial & $\begin{array}{l}0450766(\mathrm{X}) \\
4425763(\mathrm{Y})\end{array}$ \\
\hline 2 & $\begin{array}{l}\text { Haplic Luvisol } \\
\text { (Chromic, Profondic) }\end{array}$ & $\begin{array}{l}\text { Madridejos } \\
\text { (Toledo) }\end{array}$ & Margas & $\begin{array}{l}\text { Cultivos } \\
\text { de secano }\end{array}$ & $\begin{array}{l}304534(\mathrm{X}) \\
4370675(\mathrm{Y})\end{array}$ \\
\hline 3 & $\begin{array}{l}\text { Vertic Luvisol } \\
\text { (Profondic, Rhodic) }\end{array}$ & $\begin{array}{l}\text { La Solana } \\
\text { (Ciudad Real) }\end{array}$ & Arcillitas & $\begin{array}{l}\text { Cultivos } \\
\text { de secano }\end{array}$ & $\begin{array}{l}0479715(\mathrm{X}) \\
4306634(\mathrm{Y})\end{array}$ \\
\hline 4 & $\begin{array}{l}\text { Haplic Calcisol } \\
\text { (Ruptic, Chromic) }\end{array}$ & $\begin{array}{l}\text { Villahermosa } \\
\text { (Ciudad Real) }\end{array}$ & $\begin{array}{l}\text { Cuarcitas/ } \\
\text { areniscosas }\end{array}$ & Bosque & $\begin{array}{l}519091(\mathrm{X}) \\
4288625(\mathrm{Y})\end{array}$ \\
\hline 5 & $\begin{array}{l}\text { Haplic Leptosol } \\
\text { (Skeletic, Novic) }\end{array}$ & $\begin{array}{l}\text { Alcaraz } \\
\text { (Albacete) }\end{array}$ & Cuarcitas & Pastos & $\begin{array}{l}544057(\mathrm{X}) \\
4211625(\mathrm{Y})\end{array}$ \\
\hline 6 & $\begin{array}{l}\text { Cutanic Luvisol } \\
\text { (Clayic, Rhodic) }\end{array}$ & $\begin{array}{l}\text { Povedilla } \\
\text { (Albacete) }\end{array}$ & Arcillitas & $\begin{array}{l}\text { Cultivos } \\
\text { de secano }\end{array}$ & $\begin{array}{l}0540151(\mathrm{X}) \\
4282000(\mathrm{Y})\end{array}$ \\
\hline 7 & $\begin{array}{l}\text { Leptic Luvisol } \\
\text { (Humic, Chromic) }\end{array}$ & $\begin{array}{l}\text { El Bonillo } \\
\text { (Albacete) }\end{array}$ & Calizas & Bosque & $\begin{array}{l}0537754(\mathrm{X}) \\
4310260(\mathrm{Y})\end{array}$ \\
\hline 8 & $\begin{array}{l}\text { Endogleyic Cambisol } \\
\text { (Humic, Dystric) }\end{array}$ & $\begin{array}{l}\text { Velada } \\
\text { (Toledo) }\end{array}$ & Granitos & Pastos & $\begin{array}{l}331058(\mathrm{X}) \\
4431848(\mathrm{Y})\end{array}$ \\
\hline 9 & $\begin{array}{l}\text { Calcic Luvisol } \\
\text { (Chromic, Abruptic) }\end{array}$ & $\begin{array}{l}\text { Mirabueno } \\
\text { (Guadalajara) }\end{array}$ & $\begin{array}{l}\text { Calizas } \\
\text { y margas }\end{array}$ & Bosque & $\begin{array}{l}0521246(\mathrm{X}) \\
4531563(\mathrm{Y})\end{array}$ \\
\hline 10 & $\begin{array}{l}\text { Haplic Leptosol } \\
\text { (Calcaric, Skeletic) }\end{array}$ & $\begin{array}{l}\text { Torremocha } \\
\text { (Guadalajara) }\end{array}$ & $\begin{array}{l}\text { Calizas/ } \\
\text { calcarenitos }\end{array}$ & Pastos & $\begin{array}{l}0591915(\mathrm{X}) \\
4540195(\mathrm{Y})\end{array}$ \\
\hline 11 & $\begin{array}{l}\text { Leptic Cambisol } \\
\text { (Calcaric, Chromic) }\end{array}$ & $\begin{array}{l}\text { Alcolea del Pinar } \\
\text { (Guadalajara) }\end{array}$ & Calcarenitos & $\begin{array}{l}\text { Cultivos de } \\
\text { secano y pastos }\end{array}$ & $\begin{array}{l}543521(\mathrm{X}) \\
4543529(\mathrm{Y})\end{array}$ \\
\hline 12 & $\begin{array}{l}\text { Haplic Regosol } \\
\text { (Gypsirc, Calcaric) }\end{array}$ & $\begin{array}{l}\text { Tarancón } \\
\text { (Toledo) }\end{array}$ & $\begin{array}{l}\text { Margas } \\
\text { y yesos }\end{array}$ & Erial & $\begin{array}{l}0504409(\mathrm{X}) \\
4430745(\mathrm{Y}) \\
\end{array}$ \\
\hline 13 & $\begin{array}{l}\text { Cutanic Luvisol } \\
\text { (Chromic, Clayic) }\end{array}$ & $\begin{array}{l}\text { Belmonte } \\
\text { (Cuenca) }\end{array}$ & $\begin{array}{l}\text { Calizas } \\
\text { de secano }\end{array}$ & Cultivos & $\begin{array}{l}0519693(\mathrm{X}) \\
4380744(\mathrm{Y})\end{array}$ \\
\hline 14 & $\begin{array}{l}\text { Haplic Cambisol } \\
\text { (Calcaric, Yermic) }\end{array}$ & $\begin{array}{l}\text { Olivares del Júcar } \\
\text { (Cuenca) }\end{array}$ & Margas & Bosque & $\begin{array}{l}0554157(\mathrm{X}) \\
4406257(\mathrm{Y})\end{array}$ \\
\hline 15 & $\begin{array}{l}\text { Leptic Cambisol } \\
\text { (Dystric, Skeletic) }\end{array}$ & $\begin{array}{l}\text { Sta. Cruz de Mudela } \\
\text { (Ciudad Real) }\end{array}$ & $\begin{array}{l}\text { Cuarcitas } \\
\text { y pizarras }\end{array}$ & Erial & $\begin{array}{l}459873(\mathrm{X}) \\
4278354(\mathrm{Y})\end{array}$ \\
\hline 16 & $\begin{array}{l}\text { Calcic Luvisol } \\
\text { (Rhodic, Profondic) }\end{array}$ & $\begin{array}{l}\text { Bazán } \\
\text { (Ciudad Real) }\end{array}$ & $\begin{array}{l}\text { Cuarcitas } \\
\text { y pizarras }\end{array}$ & $\begin{array}{l}\text { Cultivos } \\
\text { de secano }\end{array}$ & $\begin{array}{l}0457194(\mathrm{X}) \\
4270927(\mathrm{Y})\end{array}$ \\
\hline 17 & $\begin{array}{l}\text { Calcic Vertisol } \\
\text { (Humic, Pellic) }\end{array}$ & $\begin{array}{l}\text { Valverde } \\
\text { (Ciudad Real) }\end{array}$ & Basaltos & $\begin{array}{l}\text { Cultivos } \\
\text { de secano }\end{array}$ & $\begin{array}{l}409417(\mathrm{X}) \\
4316228(\mathrm{Y})\end{array}$ \\
\hline 18 & $\begin{array}{l}\text { Calcic Luvisol } \\
\text { (Skeletic, Rhodic) }\end{array}$ & $\begin{array}{l}\text { Ocaña } \\
\text { (Toledo) }\end{array}$ & Calizas & Erial & $\begin{array}{l}455084(\mathrm{X}) \\
4423063(\mathrm{Y})\end{array}$ \\
\hline 19 & $\begin{array}{l}\text { Haplic Fluvisol } \\
\text { (Calcaric, Skeletic) }\end{array}$ & $\begin{array}{l}\text { Balazote } \\
\text { (Albacete) }\end{array}$ & $\begin{array}{l}\text { Sedimentos } \\
\text { fluviales }\end{array}$ & $\begin{array}{l}\text { Cultivos } \\
\text { de secano }\end{array}$ & $\begin{array}{l}0580442(\mathrm{X}) \\
4307137(\mathrm{Y})\end{array}$ \\
\hline 20 & $\begin{array}{l}\text { Leptic Cambisol } \\
\text { (Humic, Dystric) }\end{array}$ & $\begin{array}{l}\text { Almadén } \\
\text { (Ciudad Real) }\end{array}$ & Pizarras & $\begin{array}{l}\text { Erial/ } \\
\text { pastos }\end{array}$ & $\begin{array}{l}340446(\mathrm{X}) \\
4290941(\mathrm{Y})\end{array}$ \\
\hline 21 & $\begin{array}{l}\text { Cambic Umbrisol } \\
\text { (Humic, Arenic) }\end{array}$ & $\begin{array}{l}\text { Almorox } \\
\text { (Toledo) }\end{array}$ & Granitos & Bosque & $\begin{array}{l}0383535(\mathrm{X}) \\
4460106(\mathrm{Y})\end{array}$ \\
\hline 22 & $\begin{array}{l}\text { Haplic Acrisol } \\
\text { (Ruptic, Profondic) }\end{array}$ & $\begin{array}{l}\text { Viñuelas } \\
\text { (Guadalajara) }\end{array}$ & Rañas & Bosque & $\begin{array}{l}0470537(\mathrm{X}) \\
4515645(\mathrm{Y})\end{array}$ \\
\hline 23 & $\begin{array}{l}\text { Umbric Leptosol } \\
\text { (Humic, Dystric) }\end{array}$ & $\begin{array}{l}\text { Valdepeñas de la S. } \\
\text { (Guadalajara) }\end{array}$ & $\begin{array}{l}\text { Cuarcitas } \\
\text { y pizarras }\end{array}$ & Bosque & $\begin{array}{l}0466594(\mathrm{X}) \\
4529098(\mathrm{Y})\end{array}$ \\
\hline 24 & $\begin{array}{l}\text { Rendzic Leptosol } \\
\text { (Humic, Brunic) }\end{array}$ & $\begin{array}{l}\text { El Bonete } \\
\text { (Albacete) }\end{array}$ & Calizas & Bosque & $\begin{array}{l}0642565(\mathrm{X}) \\
4306691(\mathrm{Y})\end{array}$ \\
\hline
\end{tabular}


Tabla 2.-Valores medios de las concentraciones totales de los elementos traza, por cada horizonte (mgKg-1)

\begin{tabular}{lcccccccccc}
\hline Horizonte & $\mathrm{Sc}$ & $\mathrm{V}$ & $\mathrm{Cr}$ & $\mathrm{Co}$ & $\mathrm{Ni}$ & $\mathrm{Cu}$ & $\mathrm{Zn}$ & $\mathrm{Ga}$ & $\mathrm{Ge}^{*}$ & $\mathrm{As}$ \\
\hline $\mathrm{A}$ & 19,33 & 59,25 & 67,63 & 8,13 & 25,78 & 12,96 & 45,75 & 12,14 & 1,03 & 8,15 \\
$\mathrm{~B}-\mathrm{C}$ & 22,42 & 59,54 & 78,54 & 8,58 & 25,48 & 12,99 & 40,72 & 12,75 & 0,99 & 9,37 \\
$\mathrm{C}-\mathrm{R}$ & 27,38 & 46,80 & 56,54 & 7,63 & 20,81 & 9,89 & 32,19 & 10,43 & 0,99 & 5,74 \\
\hline Horizonte & $\mathrm{Se}^{*}$ & $\mathrm{Br}$ & $\mathrm{Rb}$ & $\mathrm{Sr}$ & $\mathrm{Y}$ & $\mathrm{Zr}$ & $\mathrm{Nb}$ & $\mathrm{Mo}^{*}$ & $\mathrm{Ag}^{*}$ & $\mathrm{Cd}^{*}$ \\
\hline $\mathrm{A}$ & 0,77 & 8,58 & 83,52 & 297,86 & 21,03 & 224,38 & 10,73 & 0,91 & 4,25 & 3,95 \\
$\mathrm{~B}-\mathrm{C}$ & 0,90 & 6,28 & 104,55 & 394,59 & 20,21 & 190,73 & 10,37 & 1,08 & 4,23 & 4,01 \\
C-R & 0,91 & 5,63 & 71,35 & 415,03 & 14,64 & 117,94 & 8,78 & 0,98 & 4,44 & 4,00 \\
\hline Horizonte & $\mathrm{Sn}$ & $\mathrm{Sb}^{*}$ & $\mathrm{I}$ & $\mathrm{Cs}$ & $\mathrm{Ba}$ & $\mathrm{La}$ & $\mathrm{Ce}$ & $\mathrm{Nd}$ & $\mathrm{Sm}^{*}$ & $\mathrm{Yb}$ \\
\hline A & 4,29 & 2,91 & 6,35 & 7,47 & 415,32 & 27,85 & 63,90 & 25,51 & 7,28 & 2,83 \\
B-C & 7,25 & 3,10 & 6,86 & 8,24 & 401,81 & 26,96 & 62,53 & 24,07 & 7,14 & 2,73 \\
C-R & 3,64 & 2,76 & 8,76 & 7,28 & 409,63 & 20,70 & 53,69 & 19,73 & 6,80 & 2,89 \\
\hline Horizonte & $\mathrm{Hf}$ & $\mathrm{Ta}^{*}$ & $\mathrm{~W}$ & $\mathrm{Tl}$ & $\mathrm{Pb}$ & $\mathrm{Bi}$ & $\mathrm{Th}$ & $\mathrm{U}$ & & \\
\hline A & 5,82 & 2,17 & 3,44 & 1,37 & 27,58 & 1,12 & 10,86 & 3,66 & & \\
B-C & 5,11 & 2,30 & 3,44 & 1,54 & 22,26 & 1,30 & 10,52 & 3,94 & &
\end{tabular}

Estimación preliminar (bajo límite de detección).

Tabla 3.-Factores de enriquecimiento (F.E.) para los distintos elementos traza

\begin{tabular}{lcccccccccc}
\hline F.E. & $\mathrm{Sc}$ & $\mathrm{V}$ & $\mathrm{Cr}$ & $\mathrm{Co}$ & $\mathrm{Ni}$ & $\mathrm{Cu}$ & $\mathrm{Zn}$ & $\mathrm{Ga}$ & $\mathrm{Ge}$ & $\mathrm{As}$ \\
\hline (A/C) & 0,71 & 1,27 & 1,20 & 1,07 & 1,24 & 1,31 & 1,42 & 1,16 & 1,04 & 1,42 \\
(B/C) & 0,82 & 1,27 & 1,39 & 1,12 & 1,22 & 1,31 & 1,26 & 1,22 & 1,00 & 1,63 \\
(A/B) & 0,86 & 1,00 & 0,86 & 0,95 & 1,01 & 1,00 & 1,12 & 0,95 & 1,04 & 0,87 \\
\hline F.E. & $\mathrm{Se}$ & $\mathrm{Br}$ & $\mathrm{Rb}$ & $\mathrm{Sr}$ & $\mathrm{Y}$ & $\mathrm{Zr}$ & $\mathrm{Nb}$ & $\mathrm{Mo}$ & $\mathrm{Ag}$ & $\mathrm{Cd}$ \\
\hline (A/C) & 0,84 & 1,52 & 1,17 & 0,72 & 1,44 & 1,90 & 1,22 & 0,93 & 0,96 & 0,99 \\
(B/C) & 1,00 & 1,12 & 1,47 & 0,95 & 1,38 & 1,62 & 1,18 & 1,10 & 0,95 & 1,00 \\
(A/B) & 0,85 & 1,37 & 0,80 & 0,75 & 1,04 & 1,18 & 1,04 & 0,85 & 1,00 & 0,99 \\
\hline F.E. & $\mathrm{Sn}$ & $\mathrm{Sb}$ & $\mathrm{I}$ & $\mathrm{Cs}$ & $\mathrm{Ba}$ & $\mathrm{La}$ & $\mathrm{Ce}$ & $\mathrm{Nd}$ & $\mathrm{Sm}$ & $\mathrm{Yb}$ \\
\hline (A/C) & 1,18 & 1,06 & 0,73 & 1,03 & 1,01 & 1,35 & 1,19 & 1,29 & 1,07 & 0,98 \\
(B/C) & 1,99 & 1,12 & 0,78 & 1,13 & 0,98 & 1,30 & 1,16 & 1,22 & 1,05 & 0,95 \\
(A/B) & 0,59 & 0,94 & 0,93 & 0,91 & 1,03 & 1,03 & 1,02 & 1,06 & 1,02 & 1,03 \\
\hline F.E. & $\mathrm{Hf}$ & $\mathrm{Ta}$ & $\mathrm{W}$ & $\mathrm{Tl}$ & $\mathrm{Pb}$ & $\mathrm{Bi}$ & $\mathrm{Th}$ & $\mathrm{U}$ & & \\
\hline (A/C) & 1,37 & 0,90 & 1,15 & 0,90 & 2,26 & 0,89 & 1,38 & 0,97 & & \\
(B/C) & 1,20 & 0,95 & 1,15 & 1,01 & 1,82 & 1,03 & 1,34 & 1,05 & & \\
(A/B) & 1,14 & 0,94 & 1,00 & 0,89 & 1,24 & 0,86 & 1,03 & 0,93 & & \\
\hline
\end{tabular}

mentos son poco apreciables, de forma que los horizontes del suelo en realidad tienen su propia línea base de concentración geoquímica. De esta forma, la variada composición mineral de las rocas, es la responsable de las diferencias en la variación de las concentraciones de los elementos.

Así pues, las concentraciones de elementos traza en Castilla-La Mancha están controladas por las características geoquímicas de la roca subyacente, siendo de menor importancia la naturaleza de los procesos edáficos, debido a la naturaleza mayorita- riamente calcárea y el bajo valor de las razones precipitación/evaporación; es decir, el medio ambiente semiárido proporciona las condiciones óptimas para que las concentraciones de elementos traza dependan en menor escala de los procesos edáficos.

La concentración media de cada elemento muestra variaciones, por lo que a veces el enriquecimiento es en la superficie, otras veces en profundidad y en ocasiones en el horizonte de profundidad intermedia (factor de enriquecimiento de la tabla 3). Por último, algunos elementos ni aumentan ni disminu- 
Tabla 4.-Calidad de suelos. Fondos geoquímicos, edafogeoquímicos y valores de referencia de 38 elementos trazas $\left(\mathrm{mgkg}^{-1}\right)$ en Castilla-La Mancha

\begin{tabular}{|c|c|c|c|c|}
\hline $\begin{array}{l}\text { Elemento } \\
\text { traza }\end{array}$ & $\begin{array}{l}\text { Fondo } \\
\text { geoquímico }\end{array}$ & $\begin{array}{c}\text { Valor } \\
\text { edafogeoquímico }\end{array}$ & $\begin{array}{c}\text { Desviación } \\
\text { estándar }\end{array}$ & $\begin{array}{l}\text { Valor de } \\
\text { referencia }\end{array}$ \\
\hline $\mathrm{Sc}$ & 27,38 & 23,6 & 13,5 & 50,8 \\
\hline V & 46,80 & 49,9 & 36,6 & 123,2 \\
\hline $\mathrm{Cr}$ & 56,54 & 54,8 & 29,2 & 113,4 \\
\hline $\mathrm{Co}^{*}$ & 7,63 & 5,8 & 7,4 & 20,8 \\
\hline $\mathrm{Ni}$ & 20,81 & 16,9 & 12,8 & 42,6 \\
\hline $\mathrm{Cu}$ & 9,89 & 10,3 & 8,3 & 27,0 \\
\hline $\mathrm{Zn}$ & 32,19 & 35,7 & 25,4 & 86,5 \\
\hline $\mathrm{Ga}$ & 10,43 & 11,1 & 7,7 & 26,7 \\
\hline $\mathrm{Ge}^{*}$ & 0,99 & 0,9 & 0,1 & 1,3 \\
\hline As & 5,74 & 7,4 & 4,2 & 16,1 \\
\hline $\mathrm{Se}^{*}$ & 0,91 & 0,8 & 0,2 & 1,4 \\
\hline $\mathrm{Br}$ & 5,63 & 6,7 & 6,6 & 20,1 \\
\hline $\mathrm{Rb}$ & 71,35 & 86,2 & 74,2 & 234,7 \\
\hline $\mathrm{Sr}$ & 415,03 & 380,0 & 744,2 & $1.868,4$ \\
\hline$Y$ & 14,64 & 17,9 & 10,1 & 38,3 \\
\hline $\mathrm{Zr}$ & 117,94 & 167,6 & 122,7 & 413,1 \\
\hline $\mathrm{Nb}$ & 8,78 & 8,0 & 5,3 & 18,7 \\
\hline Mo* & 0,98 & 0,9 & 0,4 & 2,0 \\
\hline $\mathrm{Ag}^{*}$ & 4,44 & 4,3 & 1,7 & 7,8 \\
\hline $\mathrm{Cd}^{*}$ & 4,00 & 3,9 & 0,1 & 4,4 \\
\hline $\mathrm{Sn}$ & 3,64 & 4,0 & 2,2 & 8,7 \\
\hline $\mathrm{Sb}^{*}$ & 2,76 & 2,7 & 1,4 & 5,7 \\
\hline I & 8,76 & 7,4 & 8,9 & 25,4 \\
\hline Cs & 7,28 & 7,7 & 3,2 & 14,2 \\
\hline $\mathrm{Ba}$ & 409,63 & 389,9 & 329,6 & $1.049,3$ \\
\hline $\mathrm{La}$ & 20,70 & 23,6 & 12,3 & 48,4 \\
\hline $\mathrm{Ce}$ & 53,69 & 57,7 & 20,0 & 97,9 \\
\hline $\mathrm{Nd}$ & 19,73 & 21,6 & 9,2 & 40,1 \\
\hline $\mathrm{Sm}^{*}$ & 6,80 & 6,9 & 1,8 & 10,7 \\
\hline $\mathrm{Yb}^{*}$ & 2,89 & 2,7 & 0,6 & 4,2 \\
\hline Hf & 4,24 & 4,9 & 2,5 & 10,0 \\
\hline $\mathrm{Ta}^{*}$ & 2,41 & 2,2 & 0,8 & 4,0 \\
\hline W & 2,99 & 3,2 & 1,1 & 5,5 \\
\hline $\mathrm{Tl}^{*}$ & 1,52 & 1,4 & 0,4 & 2,3 \\
\hline $\mathrm{Pb}$ & 12,20 & 19,3 & 12,3 & 44,2 \\
\hline $\mathrm{Bi}^{*}$ & 1,25 & 1,2 & 0,4 & 2,2 \\
\hline Th & 7,85 & 9,6 & 6,0 & 21,6 \\
\hline U & 3,77 & 3,8 & 3,2 & 10,3 \\
\hline
\end{tabular}

Estimación preliminar (bajo límite de detección).

yen de manera sensible su concentración con la profundidad. Así, el Sc tiene ligera tendencia a aumentar con la profundidad. El V tiende a aumentar en superficie (el mayor contenido se puede encontrar en el perfil de 17 Valverde, desarrollado en basaltos). En este mismo suelo (Vertisol), el Cr también está en alta concentración. Tienden a aumentar en superficie $\mathrm{Ni}, \mathrm{Cu}, \mathrm{As}, \mathrm{Ga}$ y también el $\mathrm{Zn}$, aunque este último a veces disminuye. En el perfil 15 (Sta. Cruz de Mudela, en las pizarras y cuarcitas), este elemento aparece en grandes cantidades. El Rb, que tiende a aumentar con la profundidad, aparece en alto contenido en el perfil 8 (Velada) sobre el sustrato granítico. El Sr aparece en una amplia gama de valores, con un alto contenido en los perfiles 1 (Ontígola) y 12 (Tarancón), desarrollados sobre margas y yesos. El Se se encuentra bajo el límite de detección; sin embargo, aunque no hemos encontrado suelos seleníferos en la región, Moreno et al. (2005) citan que se pueden encontrar en zonas de la Comunidad de Madrid.

La reciente publicación del Real Decreto 9/2005 (BOE de 2005) es un intento de limitar la diferencia de criterios. Cuando la distribución es normal (como es el caso en la mayoría de los elementos traza de los suelos estudiados) se utiliza la media aritmética. El límite superior del valor de fondo o valor de referencia se determinará mediante la fórmula VR $=\mathrm{VF}+2 \mathrm{DS}$, donde VF es el valor de fondo a partir de la media y DS la desviación estándar. Conde et al. (2009) analizaron los mismos suelos y observaron que no hubo diferencia significativa entre los horizontes superficiales y subsuperficiales, proponiendo que la estimación de los «niveles de referencia genérica» se realice indistintamente a través de cualquiera de ellos. A la luz de la hipótesis elegida, se proponen, de forma preliminar, los valores edafogeoquímicos de concentración total de elementos traza en Castilla-La Mancha que aparecen en la tabla 4. Valores de referencia que proporcionan información necesaria para establecer los criterios para declarar si un suelo está contaminado.

Este trabajo no habría podido llevarse a cabo sin el conocimiento de los suelos, su formación, su morfología y su funcionamiento, experiencia obtenida de numerosos trabajos realizados en la región por nosotros mismos. Los valores de referencia aportados (tabla 4) deben proporcionar una guía para evaluar la calidad del suelo y para ayudar a establecer el valor límite máximo de los elementos traza en suelos de Castilla-La Mancha. Esto debería evitar utilizar valores normativos de la legislación medioambiental copiada de otros países o regiones.

\section{Conclusiones}

A pesar del moderado número de muestras tomadas, el enfoque y los datos aportados permiten determinar los fondos geoquímicos y los niveles edafogeoquímicos de base, así como los valores de referencia en Castilla-La Mancha.

Todos los elementos traza muestran concentraciones promedio similares con los valores de referencia para los suelos de otras zonas de España (Galicia, 
Andalucía, Madrid, etc.), la Unión Europea y el mundo. Los patrones de distribución de las concentraciones de los elementos son principalmente influenciados por la naturaleza geoquímica de la roca.

Todos estos valores permiten hacer la evaluación de una posible contaminación. Sin embargo, la limitación del número de perfiles muestreados genera cierta incertidumbre, sobre todo en lo que se refiere a la representación espacial.

\section{Referencias}

Albanese, S.; De Vivo, B.; Lima, B. \& Cicchella, D. (2007). Geochemical background and baseline values of toxic elements in stream sediments of Campania region (Italy), Journal of Geochemical Exploration, 93: 21-34. doi:10.1016/j.gexplo.2006.07.006

Bak, J.; Jensen, J.; Larsen, M.M.; Pritzl, G. \& Scott-Fordsmand, J. (1997). A heavy metal monitoring-programme in Denmark. The Science of the Total Environment, 207: 179-186. doi:10.1016/S0048-9697(97)00262-3

Baize, D. \& Sterckeman, T. (2001). Of the necessity of knowledge of the natural Pedo-geochemical background content of soils by trace elements. The Science of the Total Environment, 264: 127-139. doi:10.1016/S0048-9697(00)00615-X

BOE (2005). Real Decreto 9/2005. Boletín Oficial del Estado BOE, $\mathrm{n}^{\mathrm{o}}$ 15, de 18 de enero de 2005. Madrid.

Cicchella, D.; De Vivo, B. \& Lima, A. (2005). Background and baseline concentration values of elements harmful to human health in the volcanic soils of the metropolitan provincial area of Napoli (Italy), Geochemistry: Exploration, Environment, Analysis, 5: 29-40. doi:10.1144/1467-7873/03-042

Conde, P.; Martín Rubí, J.A.; De la Horra, J. \& JiménezBallesta, R. (2009). Spatial and vertical distribution of trace elements in different soils of a Mediterranean environment: Castilla-La Mancha, Spain. Fresenius Environmental Bulletin, 18: 858-867.

Conde, P.; Bellido, E.; Martín Rubí, J.A. \& Jiménez Ballesta, R. (2008). Concentration and spatial variability of mercury and other heavy metals in surface soil samples of periurban waste mine tailling along a transect in the Almadén mining district (Spain). Environmental Geology, 56: 815-824.

doi: 10.1007/s00254-007-1182-z

Chen, M.; Lena, Q.M. \& Harris, W.G. (1999). Baseline concentrations of 15 trace elements in Florida surface soils. Journal of Environmental Quality, 28: 1173-1181.

De Miguel, E.; Callaba, A.; Arranz, J.C.; Cala, V.; Chacón, E.; Gallego, E.; Alberuche, E.; Alonso, C.; Fernández-Canteli, P.; Iribarren, I. \& Palacios, H. (2002). Determinación de niveles de fondo y niveles de referencia de metales pesados y otros elementos traza en suelos de la Comunidad de Madrid. Serie Medio Ambiente, Terrenos contaminados, $\mathrm{n}^{\mathrm{o}}$ 2, Instituto Geológico y Minero de España, Madrid, 167 pp.
Del Castillo, M.; Martín Rubí, J.A. \& Jiménez Ballesta, R. (2003). Estándares de calidad de los suelos del Campo de Montiel. Edafología, 9-3: 295-304.

FAO, ISRIC, ISSS (2006). World reference base for soil resources. A framework for international classification, correlation and communication. World soil resources reports 103, Rome, $132 \mathrm{pp}$.

Ferguson, L. (1989). The Heavy Elements: Chemistry, Environmental Impact and Health Effects. Pergamon Press, Oxford, 614 pp.

Fleischhauer, H.L. \& Korte, N. (1990). Formulation of cleanup standarts for trace elements with probability plots. Environmental Management, 14: 95-105. doi:10.1007/BF02394023

Frattini, P.; De Vivo, B.; Lima, A. \& Cicchella, D. (2006). Background and baseline values of human health harmful elements and gamma-ray survey in the volcanic soils of Ischia island (Italy), Geochemistry: Exploration, Environment, Analysis, 6: 325-339. doi:10.1144/1467-7873/06-105

IHOBE (1998). Valores indicativos de evaluación (VIE-A, $V I E-B$ y VIE-C) para la investigación de la contaminación del suelo. Sociedad Pública de Gestión Ambiental, Departamento de Urbanismo, Vivienda y Medio Ambiente, País Vasco, 5-45.

Junta de Andalucía (1999). Los criterios estándares para declarar un suelo contaminado en Andalucía y la metodología y técnicas de toma de muestras y análisis para su investigación. Consejería de Medio Ambiente de Junta de Andalucía, 253 pp.

Junta de Castilla y León (1999). Estudio piloto para la determinación de los niveles de fondo y valores de referencia en los suelos de Valladolid. Junta de Castilla y León.

Junta de Residus (1998). Estudi sobre els valors de fons dels sóls situats en l'ambit industrial de Catalunya. Departament de Medi Ambient, Generalitat de Catalunya.

Kabata-Pendias, A.; Dudka, S.; Chlopecka, A. \& Gawinowska, T. (1984). Background levels and environmental influences on trace metals in soils of the temperate humid zone of Europe. In: Biogeochemistry of trace metals (Adriano, D.C., ed.), Lewis Publishers, Boca Raton, 61-84.

Kabata-Pendias, A. \& Pendias, H. (1992). Trace elements in soils and plants. $2^{\text {nd }}$ ed. CRC Press, Boca Raton, Florida, 365 pp.

Lee, B.D.; Carter, B.J.; Basta, N.T. \& Weaver, B (1997). Factors influencing heavy metal distribution in six Oklahoma Benchmark soils. Soil Science Society of America Journal, 61: 218-223.

Ma, L.Q.; Tan, F. \& Harris, W.G. (1997). Concentrations and distributions of eleven metals in Florida soils. Journal of Environmental Quality, 26: 769-775.

Macías, F. \& Calvo de Anta, R. (2009). Niveles genéricos de referencia de metales pesados y otros elementos traza en suelos de Galicia. Xunta de Galicia. 229 pp.

Martínez-Sánchez, M.J. \& Pérez-Sirvent, C. (2008). Niveles de fondo y niveles genéricos de referencia de metales pesados en suelos de la Región de Murcia. 
Consejería de Desarrollo sotenible y Ordenación del territorio, Murcia.

McKeague, J.A. \& Wolynetz, M.S. (1980). Background levels of minor elements in some Canadian soils. Geoderma, 24: 299-307. doi:10.1016/0016-7061(80)90057-9

Moreno, M.J.; Cala, V. \& Jiménez-Ballesta, R. (2005). Selenium distribution in topsoils and plants of a semiarid Mediterranean environment. Environmental Geochemistry and Health, 27: 513-519. doi:10.1007/s10653-005-8625-9

Nsouli, B.; Darwish, T.; Thomas, J.P.; Zahraman, K. \& Roumié, M. (2004). Ni, $\mathrm{Cu}, \mathrm{Zn}$ and $\mathrm{Pb}$ background values determination in representative Lebanese soil using the thick target PIXE technique. Nuclear Instruments and Methods in Physics Research B, 119-220: 181-186.

Reimann, C. \& Garrett, R.G. (2005). Geochemical background. Concept and reality. Science of the Total Environment, 350: 12-27. doi:10.1016/j.scitotenv.2005.01.047

Reimann, C.; Filzmoser, P. \& Garrett, R.G. (2005). Background and threshold: critical comparison of methods of determination. Science of the Total Environment, 346: 1-16. doi:10.1016/j.scitotenv.2004.11.023

Salminen, R. \& Tarvainen, T. (1997). The problem of defining geochemical baselines. A case study of selected elements and geological materials in Finland. Journal of Geochemical Exploration, 60: 91-98. doi:10.1016/S0375-6742(97)00028-9

Salminen, R. \& Gregorauskiene, G. (2000). Considerations regarding the definition of a geochemical baseline of elements in the surficial materials in areas differing in basic geology. Applied Geochemistry, 15: 647-653. doi:10.1016/S0883-2927(99)00077-3

Sierra, M.; Martínez, F.J. \& Aguilar, J. (2007). Baselines for trace elements and evaluation of environmental risk in soils of Almería (SE Spain). Geoderma, 139: 209-219. doi:10.1016/j.geoderma.2007.02.003

Soil Survey Staff (2006). Keys to Soil Taxonomy. Tenth edition. NRCS. USDA. 332 pp.

Tack, F.M.G.; Verloo, M.G.; Vanmechelen, L. \& Van Ranst, E. (1997). Baseline concentration levels of trace elements as a function of clay and organic carbon contents in soils in Flanders (Belgium). Science of the Total Environment, 201: 113-123. doi:10.1016/S0048-9697(97)00096-X

Tarvainen, T. \& Kallio, E. (2002). Baselines of certain bioavailable and total heavy metal concentrations in Finland. Applied Geochemistry, 17: 975-980. doi:10.1016/S08832927(02)00003-3

Thornton, I. \& Webb, J.S. (1981). Regional distribution of trace element problems in Great Britain. In: Applied Soil Trace Elements (Davies, B.E., ed.), John Wiley and Sons, Chichester, 381-439.

Vidal, J.; Pérez-Sirvent, C.; Martínez-Sánchez, M.J. \& Navarro, M.C. (2004). Origin and behaviour of heavy metals in agricultural Calcaric Fluvisols in semiarid conditions, Geoderma, 121: 257-270. doi:10.1016/j.geoderma.2003.12.001

Recibido el 5 de febrero de 2010 Aceptado el 6 de abril de 2010 Publicado online el 11 de mayo de 2010 